\title{
Science case for VLT-Planet Finder
}

\author{
C. Moutou ${ }^{1}$, J.-L. Beuzit ${ }^{2}$, R. Gratton ${ }^{3}$, D. Mouillet ${ }^{4}$, F. Allard ${ }^{5}$, \\ J.-C. Augereau ${ }^{2}$, A. Boccaletti ${ }^{6}$, S. Desidera ${ }^{3}$, M. Feldt ${ }^{7}$, \\ Th. Henning ${ }^{7}$, A.-M. Lagrange ${ }^{2}$, A. Longmore ${ }^{8}$, F. Ménard ${ }^{2}$, \\ H.-M. Schmid ${ }^{9}$, D.M. Stam ${ }^{10}$, S. Udry ${ }^{11}$ and R. Waters ${ }^{10}$ \\ ${ }^{1}$ LAM, Marseille, France, email: claire.moutou@oamp.fr \\ ${ }^{2}$ LAOG, Grenoble, France \\ ${ }^{3}$ INAF-OPD, Padova, Italy \\ ${ }^{4}$ Obs. Midi-Pyrénées, Tarbes, France \\ ${ }^{5}$ ENS, Lyon, France \\ ${ }^{6}$ Obs. Paris Meudon, France \\ ${ }^{7}$ MPIA, Heidelberg, Germany \\ ${ }^{8}$ ATC, Edimburg, UK \\ ${ }^{9} \mathrm{ETH}$, Zürich, Switzerland \\ ${ }^{10}$ UVA, Amsterdam, The Netherlands \\ ${ }^{11}$ Obs. Genève, Switzerland
}

\begin{abstract}
This paper presents the scientific case for a next generation adaptive optics instrument at the VLT, temporarily named "Planet Finder", that is aimed at detecting and characterizing extrasolar planets through the direct analysis of their emitted photons in the visible and at near-IR wavelengths. We discuss the observational niche of such an instrument to have first light in 2010, in complement to other planet search methods. To improve the efficiency (and consistency) of the search for planets with the PF, the observations will need to be organized in the form of an extensive survey of hundreds of nearby stars, predicted outputs of which are also described here. This summarizes the study phase of the instrument, conducted by two competitive teams and the recent merging of both studies, regarding the scientific impact of Planet Finder.
\end{abstract}

Keywords. stars: low-mass, brown dwarfs, planetary systems, protoplanetary disks; techniques: high angular resolution; surveys.

\section{Introduction}

On the road towards Extremely Large Telescopes and space interferometers, one needs to progressively gain orders of magnitude in contrast in the close vicinity of bright stars. This is the motivation for a near-future (2010) near-infrared, extreme adaptive optics instrument on the VLT, equipped with high-performance tools for the removal of coherent and incoherent starlight: interferential coronagraphs and differential imaging. The instrumental design and critical sub-systems are described in Beuzit et al., Schmid et al. and Fusco et al. (this volume). The current design of the VLT-Planet Finder (PF) instrument is the result of merging two independent instrumental studies. The scientific rationale on which this design was based is also the combined effort of two independent scientific groups. In the following, we discuss the observational niche and potential impact of VLT-PF, the survey strategy and its expected output. 

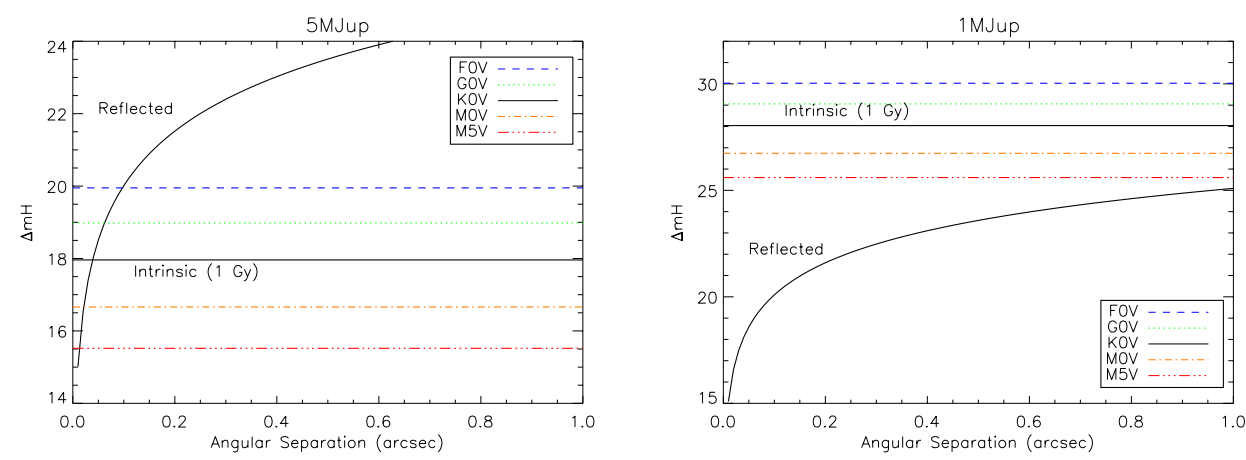

Figure 1. H-band planet-to-star flux ratio in both regimes of planet emission: intrinsic light (horizontal lines) and reflected light, for a system at 10 pc. Left: for a $5 M_{\mathrm{J}}$ of $1 \mathrm{~Gy}$. Right: for a $1 M_{\mathrm{J}}$ of $1 \mathrm{~Gy}$. The star spectral type induces more than 4 magnitudes variations in contrast, from F0V to M5V, while the reflected light drops by orders of magnitude with increasing orbital distance. The planet radius does not change much in this mass regime; here, $1 R_{\mathrm{J}}$ is used.

\section{Planet finding with VLT-PF}

At the time when VLT-PF will be in operation (2010), our knowledge of extrasolar planets will have increased significantly: the mass function and period distribution of planets will have been largely extended by ongoing radial velocity surveys, many planet radii will have been measured by transit observations, direct imaging of some very young planets will have been performed by current high contrast facilities such as NACO (see e.g. Chauvin et al. 2005, Close et al. 2004). Atmospheric models of giant planets will then have been partly constrained by observations. However, two large sets of planets will probably not be known before 2010: 1) long period planets (unaccessible to indirect methods which require a full orbit coverage), and 2) moderately young to evolved planetary systems (for which the contrast currently achieved in direct imaging is not sufficient). VLT-PF will then enlarge our view on extrasolar planetary systems, in good complementarity to other detection techniques.

When considering direct imaging of extrasolar planets, one must have in mind both types of emission that can be detected:

- Intrinsic emission of the planets, due to residual cooling after their formation. It decreases with decreasing planet mass, increasing system age. The star-planet flux ratio of this emission is independent of the orbital distance (since the planet is self-luminous) but strongly dependent on the star spectral type (later types being more favourable). Due to the planet effective temperature $(100-1000 \mathrm{~K})$, the intrinsic emission increases towards the mid-infrared. It is also characterized by spectral features, mostly due to $\mathrm{CH}_{4}$ and $\mathrm{H}_{2} \mathrm{O}$ absorption bands, and thus commonly found in spectra over an extended mass range, from Saturn mass up to brown dwarfs $\left(0.3-30 M_{\mathrm{J}}\right)$. Simulated spectra and calculated luminosities from Allard et al. (2001), Chabrier et al. (2000) and Baraffe et al. (2003) were used to estimate photon counts from the planets in this regime.

- Emission due to the reflection of starlight by the atmosphere of the planet, which strongly depends on the star-planet distance and is modulated by the phase angle. More light is reflected at wavelengths where the star is bright, i.e. in the visible, it depends on the planet radius rather than its mass, and it may be strongly polarized (see Schmid et al. this volume, and Stam et al., 2004). Physical processes taking place in the atmosphere of irradiated planets are described in Barman et al. (2001) and Sudarsky et al. (2000, 2003). 


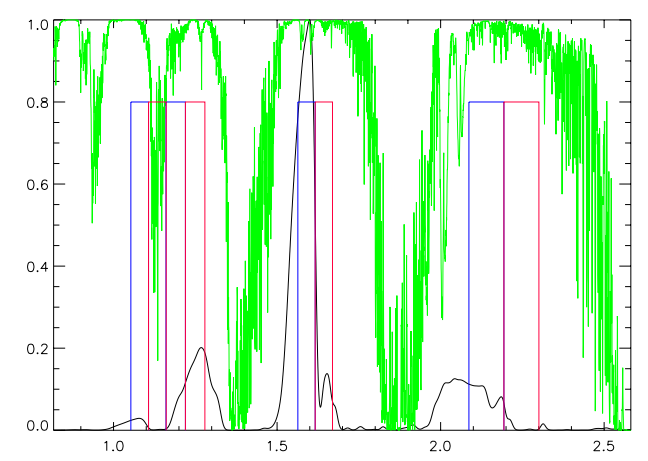

Figure 2. The spectral features of giant planets in the near-infrared ( $5 M_{\mathrm{J}}$ aged $\left.500 \mathrm{My}\right)$. In the $\mathrm{J}-$, H- and K-bands, the spectrum is characterized by deep and broad absorption bands. The filters designed for an optimal differential imaging with VLT-PF are superimposed $(\lambda / \delta \lambda \simeq 30)$, as well as the terrestrial transmission spectrum.

Figure 1 shows the relative weight of the star-planet contrast due to both types of emission, as a function of the separation. The spectral domain from $\mathrm{R}$ to $\mathrm{K}$ bands is the best compromise in terms of planet luminosity and sky background for achieving the largest contrast at short separations from the ground.

The challenge of direct imaging with VLT-PF will thus include two types of targets: those where the intrinsic light dominates (young giant planets and evolved planets in the mass regime $\simeq 10 M_{\mathrm{J}}$ ), and those where the reflected light dominates (short-period planets around the most nearby stars). VLT-PF will take advantage of the physical properties of giant planets in its detection techniques: either the spectral features or the polarisation of the planet atmosphere is used to increase the sensitivity through differential imaging methods (Beuzit et al., Schmid et al. and Boccaletti et al., this volume). Consequently, the detection of planets with VLT-PF will allow some characterization of their atmospheric components: effective temperature (depth of $\mathrm{CH}_{4}$ and $\mathrm{H}_{2} \mathrm{O}$ features, near-IR low-resolution spectro-photometry), dust grain size and cloud characteristics (degree of polarisation), planet radius and geometric albedo. VLT-PF will then mainly discover and characterize planets in the mass range 1-10 $M_{\mathrm{J}}$ and period range 1-1000 years, i.e., all gas giants that were expected before the discovery of hot jupiters. The contrast of $10^{-4}$ to $10^{-8}$ at angular separations 0.1 to 1.5 arcsec should be achieved, with a gain of up to 8 magnitudes with respect to current instruments.

\section{Survey strategy and output}

In order to assess the potential scientific output of VLT-PF, one should answer the following questions: 1) what is the detection performance of the instrument, 2) how many targets are suitable for planet search in direct imaging, 3) how do planetary and stellar characteristics combine in the detection space of VLT-PF? A mutually self-consistent detection performance of each of the VLT-PF focal instruments still has to be estimated from a unique input data set. First, independent, estimates were obtained during the competitive study phase (2002-2004) and are partly exposed in Boccaletti et al., Schmid et al. and Beuzit et al. (this volume). Table 1 summarizes some of these preliminary results. 


\begin{tabular}{|c|c|c|c|c|c|c|}
\hline Mode & Target & $0.2^{\prime \prime}$ & $0.5 "$ & $1.0 "$ & $2.0 "$ & Comment \\
\hline DBI & $\mathrm{V}=2$ & $4.010^{-6}$ & $2.510^{-6}$ & $2.510^{-6}$ & $2.010^{-6}$ & \multirow{3}{*}{$\begin{array}{l}\text { all systematics inc. } \\
\text { not all systematics inc. } \\
\text { no systematics inc. }\end{array}$} \\
\hline IFS & $\mathrm{J}=6$ & - & $10^{-7}$ & $5.010^{-8}$ & $1.210^{-8}$ & \\
\hline Zimpol & $\mathrm{I}=6$ & $6.010^{-8}$ & $3.010^{-8}$ & $2.010^{-8}$ & $1.010^{-9}$ & \\
\hline
\end{tabular}

Table 1. Contrast achieved with a SNR of 5 in 4 hours, in each mode of VLT-PF (DBI: Dual Band Imaging; IFS: Integral Field Spectrograph; Zimpol), at separations 0.2-2 arcsec, for various types of targets. Instrumental aberrations were not included in the same way in all simulations (last column). More details and consistent comparisons will be discussed in a further paper.

Three kinds of stars were identified as suitable for planet search with VLT-PF:

- The most nearby stars (3-20 pc): solar-type stars at less than 20 pc are well known and identified in Hipparcos and Gliese catalogs; M dwarfs at less than 12 pc are identified in dedicated surveys (Beuzit et al., 2004 and references therein). These targets would allow discovery of some planets through their reflected light, if their orbital distance is short enough (a few AUs typically) for this emission to significantly contribute. Around the closest $\mathrm{M}$ dwarfs, self-luminous planets could be discovered (whatever their orbital distance) since the contrast is favoured by a fainter star. Detailed detection statistics using Monte Carlo calculations and the high performance of the VLT-PF Integral Field Spectroscopy channel have revealed that evolved planets of $1 M_{\mathrm{J}}$ could be detected around stars within $20 \mathrm{pc}$, at typical contrasts of $10^{6}$. The output mass distribution of these simulations is shown in Fig. 3. The specific interest of observing the 40 closest M dwarfs accessible from the Southern hemisphere requires the adaptive optics system to operate to full specification up to $\mathrm{V}$ magnitudes of 11 if the wavefront sensing is performed in the visible (or $\mathrm{H}$ magnitude of 8 with a near-infrared sensing).

- The youngest stars (20-70 pc, 1-100 My): located in star-forming associations, these targets are of great interest since Jupiter-like planets at their earliest stage of formation and migration could be discovered. The mean distance of such associations is about 50 pc, although a few objects are known within 30 pc (see review by Zuckerman and Song, 2004). In total, there is a potential sample of about 130 stars in young associations, in the relevant magnitude range and observable from Paranal. Planets at orbital distances larger than 10 AUs will be searched for around such targets.

- Intermediate stars (3-50 pc, 0.1-1 Gy): relaxing the above constrains on the stellar age and on the distance, one may still find a large quantity of potential targets for VLT-PF. They include stars with planets known by radial velocity measurements, or stars showing a long-term drift indicative of a large distance companion. They also include active stars, that are often discarded in radial velocity surveys but whose activity may indicate a relatively young age (and hence, brighter planetary companions). In the target sample of CORALIE, up to 400 stars could have ages less than 1 Gy. Even when the age-activity relation has been calibrated statistically (Donahue 1993), there are potential additional sources of activity that mean an age estimate for an individual star using this method has considerable uncertainty.

The complementary potential of the three imaging modes of VLT-PF will allow us to probe different ages of the evolution of planetary systems, from few My to 10 Gy, providing clues for planet evolution (dynamical and atmospheric). Because the frequency of planets in the mass and period ranges accessible to PF is basically unknown, searching for planets with VLT-PF requires an extensive survey on several hundreds of stars, with a volume up to 500 nights over about 4 years. A dedicated survey will optimize the observing efficiency and ensure database consistency for 
1) perform statistical analysis of the results and 2) archive a complete data set as legacy for future missions with ELTs or space interferometers.

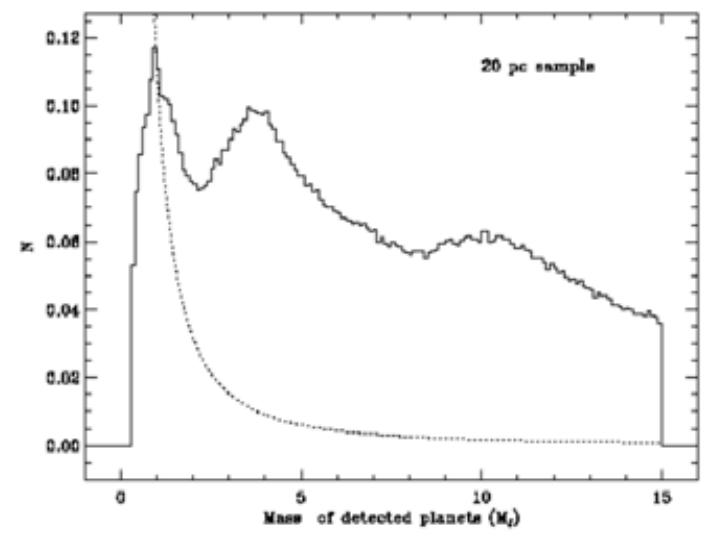

Figure 3. The simulated mass distribution of extrasolar planets that could be discovered by VLT-PF, from Monte-Carlo calculations using the performance theoretically obtained in the IFS channel. The dashed line shows the input distribution from Lineweaver \& Grether (2003).

\begin{tabular}{lllll}
\hline Type & Number & Distance & Age & $\begin{array}{l}\text { Mass, Period } \\
\text { of } 1^{s t} \text { interest planets }\end{array}$ \\
\hline Very nearby stars & 20 & $<6 \mathrm{pc}$ & $1-10 \mathrm{Gyr}$ & $1 R_{\mathrm{J}}, 0.1-10 \mathrm{y}$ \\
Nearby M stars & 40 & $<12 \mathrm{pc}$ & $1-10 \mathrm{Gyr}$ & $1-10 M_{\mathrm{J}}, 1-50 \mathrm{y}$ \\
Young associations & 130 & $20-100 \mathrm{pc}$ & $2-50 \mathrm{Myr}$ & $1-5 M_{\mathrm{J}}, 10-10^{3} \mathrm{y}$ \\
Active FGK stars & 400 & $<50 \mathrm{pc}$ & $<1 \mathrm{Gyr}$ & $5-20 M_{\mathrm{J}}, 1-50 \mathrm{y}$ \\
Stars with RV planets & $>60$ & $<50 \mathrm{pc}$ & $>1 \mathrm{Gyr}$ & $5-20 M_{\mathrm{J}}, 20-1000 \mathrm{y}$ \\
\hline Total & $\simeq 540$ & $<100 \mathrm{pc}$ & all & $1-20 M_{\mathrm{J}}, 0.1-10^{3} \mathrm{y}$ \\
\hline
\end{tabular}

Table 2. The different types of target stars suitable for planet and brown dwarf searches are listed, with their counts, distance and age ranges. The limiting magnitude is $R \simeq 10$. The typical range of planet mass and orbital period in the parameter space of VLT-PF is shown in the right column. Stars with known radial-velocity planets and active FGK stars both come from the "intermediate class of stars" described in the text. Nearby stars are separated here into spectral types, $\mathrm{M}$ dwarfs being specifically interesting nearby targets.

\section{Life of stellar and planetary systems}

With the most demanding contrast requirement of planet detection, many more science topics may be addressed by the VLT-PF instrument. Apart from marginal work on solar system objects and extragalactic sources (both being faint and extended targets, not optimal for VLT-PF and already efficiently observed with NACO-like instruments), the additional science of VLT-PF concerns the various stages of star life, from birth to death.

- Gas-rich disks: VLT-PF will allow detecting more very young, gas-rich disks around stars in Taurus or Orion clouds, and study the internal part of known disks. The polarimetric mode will be essential for characterizing grain properties in such disks.

- Young stars: physical processes during all stages of star formation will be addressed by observing jets and outflows at high contrast. 
- Debris disks: new debris disks are currently been discovered by Spitzer (Beichman et al., 2005, intrinsic disk emission); their observation with VLT-PF will provide complementary information on the light scattering properties of the disk grains from polarimetry with ZIMPOL. Disk asymmetries could be detected as possible evidence for planets.

- Evolved stars: the final stages of massive stars, characterized by mass loss, growing envelopes and shocks will be addressed by VLT-PF. Wolf-Rayet stars, novae and supernovae are potential targets.

The science topics mentioned above will benefit from the large contrast at small separations obtained by VLT-PF; some of them, however, less demanding in terms of contrast, will be used as backup programs in median atmospheric conditions.

Note that a workshop entitled "Science with Planet Finder" will be organized in the spring 2006 to help enlarge the future users' community of this instrument and to open discussion on the use of the instrument and survey for additional or alternative proposals.

\section{Conclusion}

The next generation adaptive optics instrument for the VLT, temporarily called Planet Finder, will be able of conducting a large scale survey on bright and nearby stars, in search for $10^{4}$ to $10^{8}$ times fainter companions at less than 2 arcsec. This will allow a breakthrough in our understanding of the formation and migration scenarii of giant planets and brown dwarfs, and on the physical processes at work in planetary atmospheres, at various evolution stages. VLT-PF could also contribute to understand the brown dwarf desert, if the latter is not present at early ages of the systems or if the non-detection of brown dwarfs is currently limited by sensitivity and period coverage. Gas-rich protoplanetary disks and debris disks will be observed and offer complementary information to Spitzer measurements. Polarimetric and spectroscopic facilities foreseen on VLT-PF are great tools to achieve the characterization of detected objects, that will fruitfully complement other future instruments such as JWST/MIRI.

\section{References}

Allard, F., Hauschildt, P.H., Alexander D.R. et al., 2001, ApJ 356, 557

Barman, T.S., Hauschildt, P.H. \& Allard, F., 2001, ApJ 556, 885

Baraffe, I., Chabrier, G., Barman, T.S. et al., 2003, A\&A 402, 701

Beichman, C.A., Bryden, G., Rieke, G. H. et al., 2005, ApJ 622, 1160

Beuzit, J.-L., Ségransan, D., Forveille, T. et al., 2004, A\&A 425, 997

Beuzit, J.-L. et al., 2005, this volume

Boccaletti, A. et al., 2005, this volume

Chabrier, G., Baraffe, I. Allard, F. et al., 2000, ApJ 542, 464

Chauvin G., Lagrange, A.-M., Dumas, C. et al., 2005, A\&A 438, L25

Close, L., Lenzen, R., Guirardo, J.C. et al., 2004, Nature 433, 286

Donahue, R.A., 1993, PASP 105, 804

Fusco, T. et al., 2005, this volume

Lineweaver, C.H. \& Grether, D., 2003, ApJ 598, 1350

Schmid, H.-M. et al., this volume

Stam, D.M., Hovenier, J.W. \& Waters L.B.F.M., 2004, A\&A 428, 663

Sudarsky, D., Burrows, A. \& Pinto, P., 2000, ApJ, 538, 885

Sudarsky, D., Burrows, A. \& Hubeny, I., 2003, ApJ, 588, 1121

Zuckerman, B. and Song, I., 2004, ARAA 42, 685 